\title{
How Clouds are Amplifying (or not) the Effects of ALAN
}

\author{
Salvador J. Ribas, 1,2,* Jordi Torra², Francesca Figueras², Sergi Paricio³, Ramon Canal- \\ Domingo $^{1}$ \\ ${ }^{1}$ Parc Astronòmic Montsec - Consell Comarcal de la Noguera, Àger E25691, Spain \\ ${ }^{2}$ Institut de Ciències del Cosmos (ICCUB-IEEC), Barcelona E08028, Spain \\ ${ }^{3}$ Servei per la Prevenció de la Contaminació Acústica i Lumínica (Generalitat de Catalunya), \\ Barcelona E08028, Spain
}

Received 04 December 2016, Accepted 30 December 2016

\begin{abstract}
Light emitted or reflected up to the sky can interact with clouds or fog changing Night Sky Brightness (NSB). So the evaluation of NSB in any place can be affected by this meteorological features and needs to be analyzed. To evaluate this effect, data of NSB obtained with the Catalan Light Pollution Network (XCLCat) has been analyzed accurately and shows how the effect is completely different in urban and protected areas. The study, including for the first time independent cloud coverage data from ceilometer in combination with Sky Quality Meters (SQM), shows how in a city NSB increases dramatically and in a protected area the sky appears darker than in cloudless situation.
\end{abstract}

Keywords: Environmental monitoring, Light pollution, Atmospheric effects, Photometry, Clouds

\section{Introduction}

Light Pollution (LP) is an effect generated by artificial light at night (ALAN). Normally LP is associated with astronomy, but there are many other effects in natural environment, health or power consumption [1, 2, 3, 4]. One of the most common features of LP is the emission of light to the sky, generating skyglow.

The evaluation of LP can be done measuring Night Sky brightness (NSB) using ground-based instruments as telescopes with cameras or stand-alone devices for this purpose. Light emitted or reflected up to the sky can interact with clouds or fog changing dramatically NSB (see for example [5, 6, 7]). The interaction of artificial lights with clouds can only be evaluated with ground based measurements because this interaction starts in the base of clouds layer, so any observation above the clouds will not provide clear information of this interaction. So the evaluation of NSB from the ground can be clearly affected by presence or absence of clouds. This effect is completely different depending of the nature of the site: dark site or urban polluted site.

\section{Catalan Light Pollution Network (XCLCat)}

There are different approaches to evaluate NSB to estimate LP: from the space or from the ground. The first option is based in the idea of using satellite data [8] or data from the International Space Station [9]. The ground based observations can be done using classical photometry with telescopes or using new specially designed

\footnotetext{
* Ribas SJ, Tel.: +34-973053022

E-mail address: sjribas@montsec.cat
} 
devices. In our study we have worked with ground-based measurements using Sky Quality Meter (SQM) devices. As it is explained above, ground measurements are mandatory to evaluate interaction of artificial light with clouds.

The cooperation of Catalan Service against Light Pollution and Parc Astronòmic Montsec (PAM) has leaded to a pilot plan of a network for monitoring and evaluation of NSB in different places of Catalonia region [10]. Initially the network had just five devices but in 2016 the network increased to nine devices and in the near future is expected to be integrated in the Air Quality Network (XVPCA) of the Government of Catalonia. Each station of the network is based on SQM devices, mainly SQM-LE version that works connected to Ethernet socket.

SQM devices are used pointing to zenith and also with its L (lens) versions have a wide field of view of around $40^{\circ}[11,12]$. The spectral response is close to photopic eye response but is more sensitive to blue part of visible light, so this means its response is not exactly the photopic band nor the $\mathrm{V}$ Johnson astronomical filter band. Luminances are reported in magSQM/arcsec2 but can be converted to $\mathrm{mcd} / \mathrm{m} 2$ with some assumptions (see for example $[13,14])$.

All the procedure of acquisition and preprocessing of data of XCLCat stations is done using PySQM software [15]. Currently the devices are installed with two main focuses: natural or dark sky protected areas and places that contribute to NSB of these protected sites (see Fig.1 and Table 1).

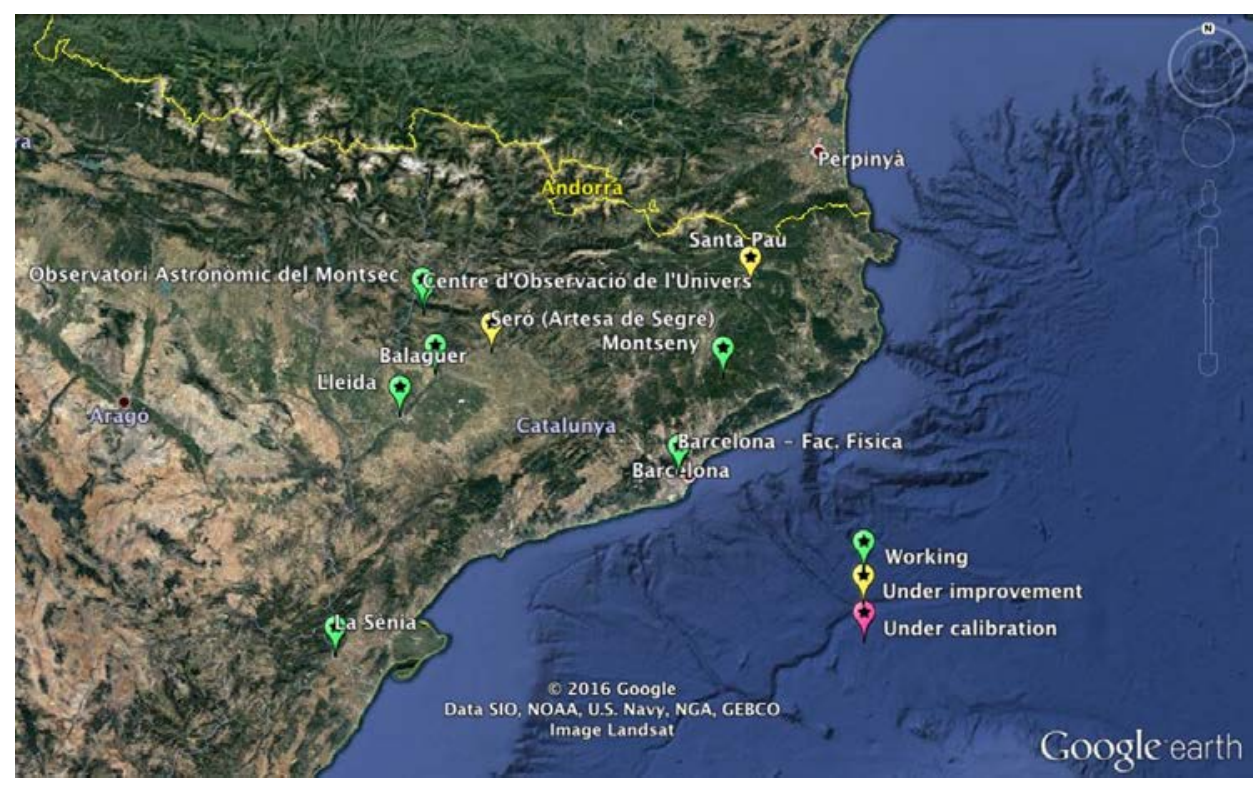

Fig. 1. Map of the current XCLCat stations (2016). The highest concentration is in STARLIGHT protected area of Montsec

Table 1. Mean Values of NSB during astronomical night in 2015 for the first five operative stations of XCLCat. It is clearly visible how in Lleida or Barcelona there is no difference when moonlit data are not included, so the lunar cycle will be poorly visible. In the other hand the first two sites listed, belonging to PAM sites, are extremely dark and the Moon effect is clear.

\begin{tabular}{|c|c|c|c|c|}
\hline Station & $\begin{array}{l}\text { GPS Position } \\
\text { Latitude \& } \\
\text { Longitude }\end{array}$ & $\begin{array}{l}\text { Population } \\
\text { (2014) }\end{array}$ & $\begin{array}{c}\text { NSB } \\
\text { Total Sample }\end{array}$ & $\begin{array}{c}\text { NSB } \\
\text { Moonless Sample }\end{array}$ \\
\hline & [deg] & [inhabitants] & [magSQM/arcsec2] & [magSQM/arcsec2] \\
\hline $\begin{array}{l}\text { Àger - Centre d'Observació } \\
\text { de l'Univers (COU) }\end{array}$ & $42.02444 \mathrm{~N} 0.73611 \mathrm{E}$ & 594 & 20.24 & 21.38 \\
\hline $\begin{array}{l}\text { Sant Esteve de la Sarga - Obs. } \\
\text { Astr. del Montsec (OAdM) }\end{array}$ & $42.05140 \mathrm{~N} 0.72962 \mathrm{E}$ & 132 & 20.25 & 21.48 \\
\hline Balaguer & $41.78681 \mathrm{~N} 0.80650 \mathrm{E}$ & 16485 & 18.13 & 18.43 \\
\hline Lleida & $41.61578 \mathrm{~N} 0.61573 \mathrm{E}$ & 139176 & 16.67 & 16.71 \\
\hline Barcelona - Fac. Física & $41.38472 \mathrm{~N} 2.11800 \mathrm{E}$ & 1602386 & 16.67 & 16.75 \\
\hline
\end{tabular}


All the instruments of the XCLCat have been intercalibrated in the specially designed station of Centre d'Observació de l'Univers. Intercalibration consists in a cross-calibration method based in pairing all the instruments, so with this strategy all the devices will show the same value for the same situation. The initial accuracy of a SQM is around $10 \%$ and with the intercalibration could be improved to $1 \%[10,16,17]$.

\section{Interaction of clouds with NSB}

One of the difficulties of compare NSB data with clouds is the quality of clouds data, sometimes only synoptic information has been used [5] and not direct measurements of clouds properties. Fortunately XCLCat has some stations that are installed in areas used for environmental studies. This is the case of Montsec Starlight protected area and the city of Barcelona. Both places have installed a ceilometer, an IR laser device that provides real time information of clouds and aerosols [18]. The ceilometer installed in Montsec is a Jenoptik CHM15K Nimbus that measures up to 14 kilometers and the ceilometer installed in Barcelona is a Vaisala CL13 that operates up to 8 kilometers [10]. So for the first time SQM and ceilometer data have been combined to evaluate the interaction of clouds and NSB, and it has been done in completely different situations of LP: dark and urban places.

The presence of clouds and fog generates oscillations in NSB values obtained with SQM's. In the absence of clouds NSB is more stable in comparison with the presence of clouds as is shown in Fig.2. In the case of cities or light polluted areas the effect of clouds is to increase the brightness of the sky [5, 6].
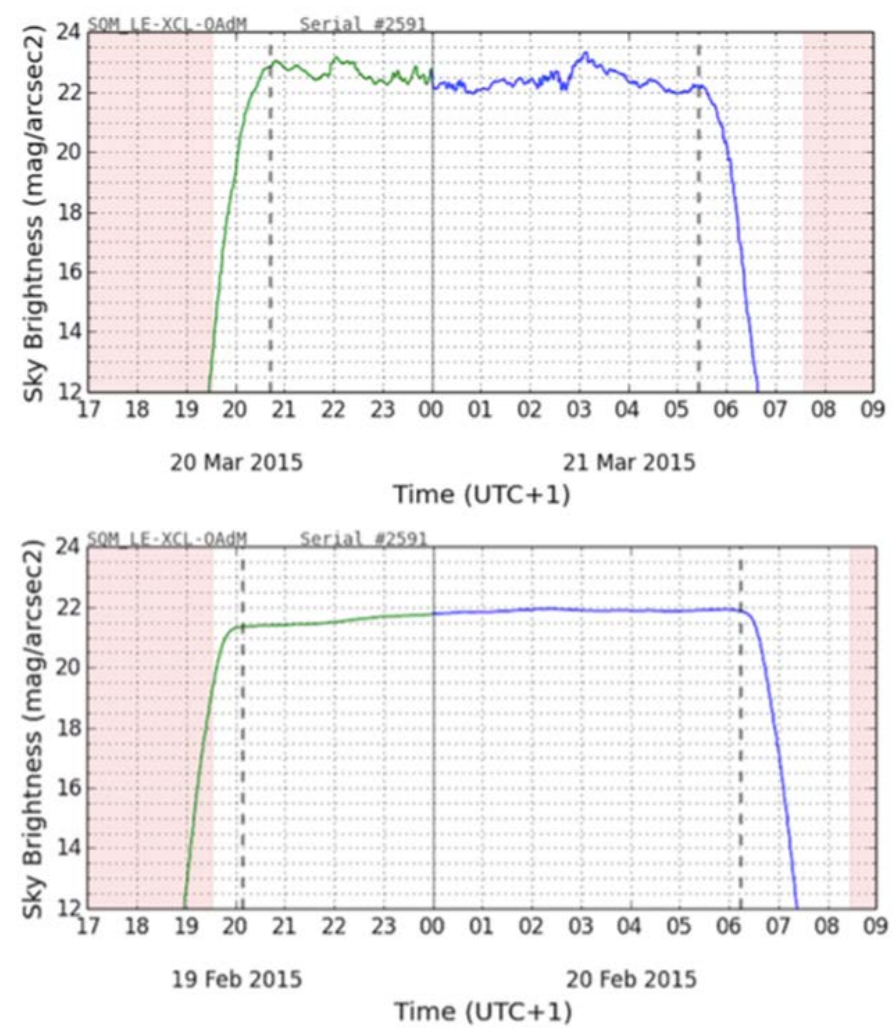

Fig. 2. Comparison of two nights of measurements with SQM. Top panel shows a night with clouds and bottom panel shows one without clouds. The night with clouds shows some oscillations on the NSB created by clouds.

The effect of increasing NSB is extremely visible in case of fog, also without the use of specific clouds data, just with evaluation of NSB in yearly plots as in Fig.3. This is the case of the cities of Balaguer, a small city with not very high LP, and Lleida, a medium size city placed $25 \mathrm{~km}$ south of Balaguer. Both cities are located in an area where it is possible to have huge episodes with permanent fog. In Fig.3 are clearly visible three periods with 
permanent fog showing an important increase of NSB up to 14-15 magnitudes per square arcsecond (red vertical stripes). It is also well identified because the red stripes (fog episodes) are completely simultaneous in both cities and could not be related to any local effect. This fog effect is not visible in the case of a site located in the mountains of Montsec, as the station placed in Observatori Astronòmic del Montsec (OAdM) at 1600 meters above sea level (asl). In Fig.4 all the lunar cycles are clearly visible as diagonal stripes and, because this site is one of the darkest in southern Europe, NSB reaches values as dark as 22 magnitudes per square arcsecond.
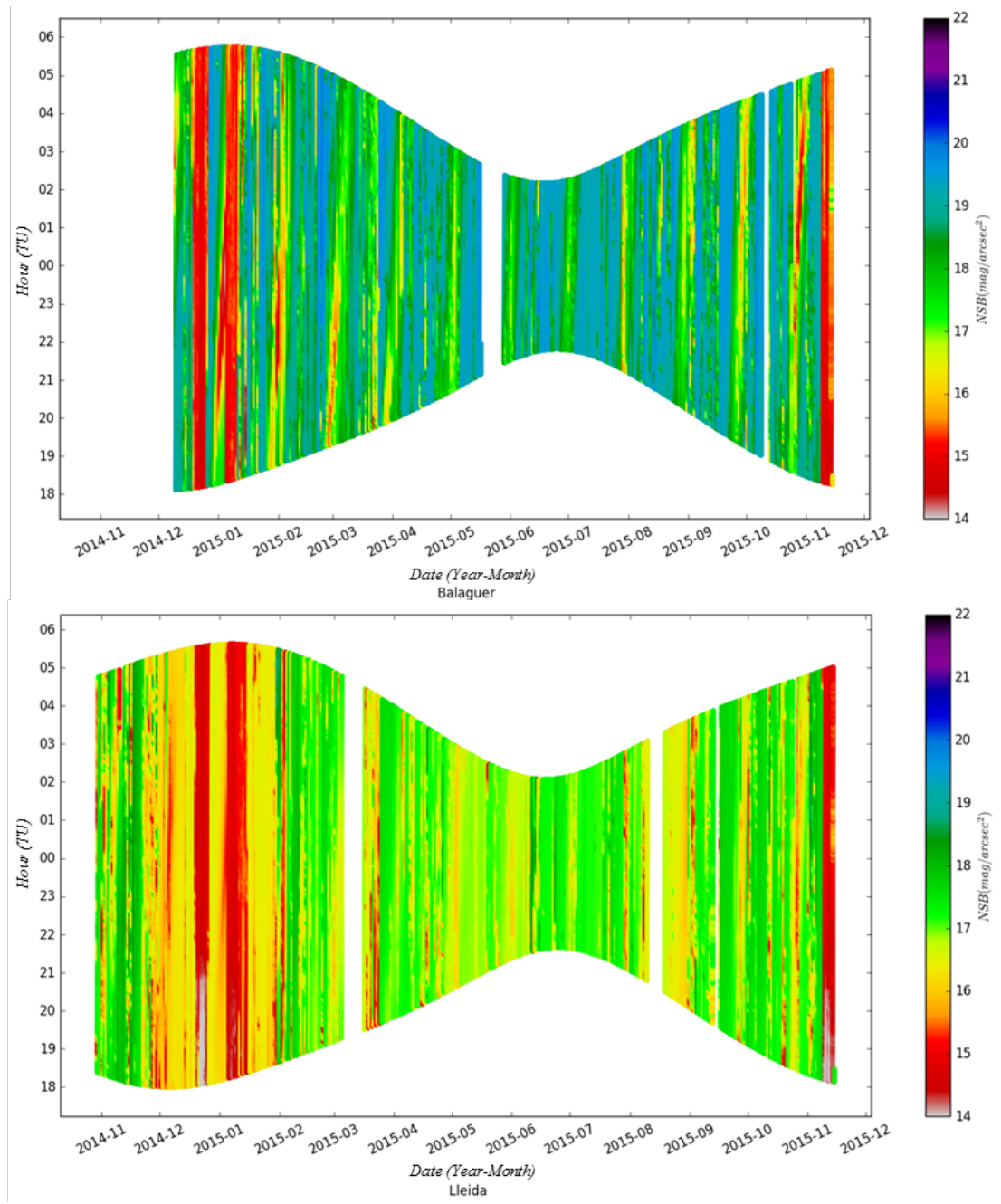

Fig. 3. Evolution of close to one year of NSB data in two sites. Top panel shows data from Balaguer and bottom panel from Lleida. In Balaguer few lunar cycles are still visible but not in Lleida. The most visible features are the three red vertical stripes related to fog episodes. 


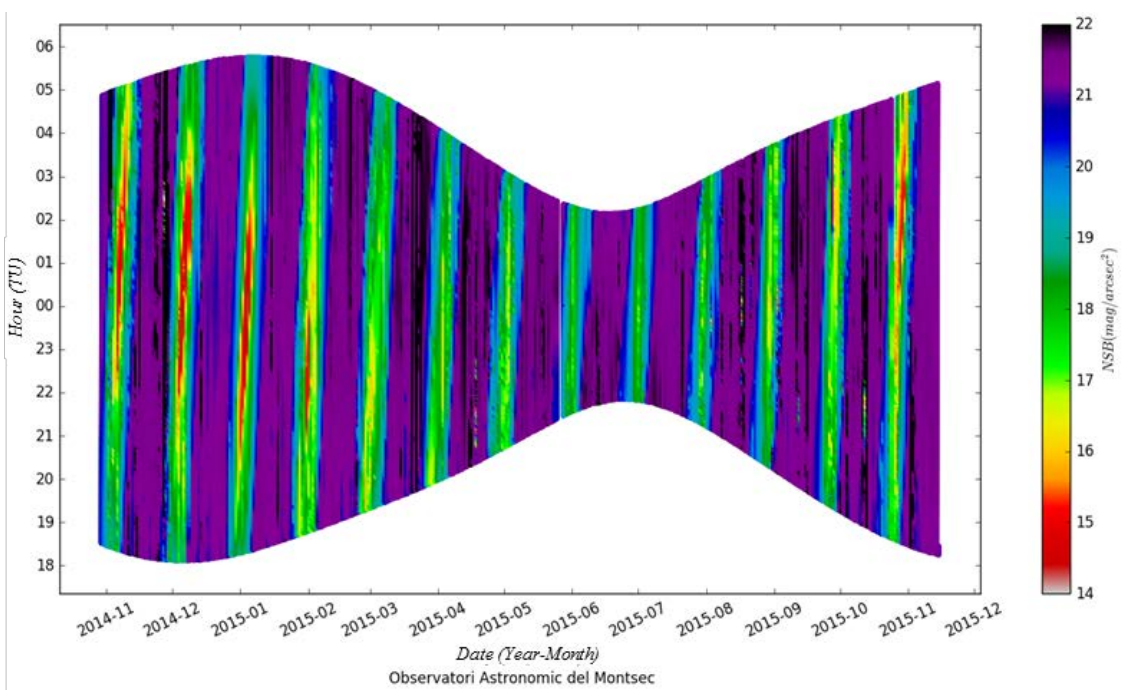

Fig. 4. Evolution of close to one year of NSB data in a very dark place, OAdM (c. $1600 \mathrm{~m}$ asl). All the lunar cycles are clearly visible as diagonal bands and there are not fog episodes in mountain location.

Another possible approach to detect clouds or fog effects is evaluating how NSB is distributed during the night, with accumulative data of one year, showing a different shape depending of the amount of LP present at the site. In Fig. 5 it is plotted the distribution of NSB in a protected dark area as OAdM site and it is clearly visible how the main distribution is around 21-22 mag and scattered measurements are moving to dark part of the plot (higher magnitudes). This scattered data could be related to measurements with clouds, so this is a first probe of clouds darkening in dark sites. This effect of darkening linked with clouds has been also recently detected with SQM data in Lake Stechlin (Germany) [7].

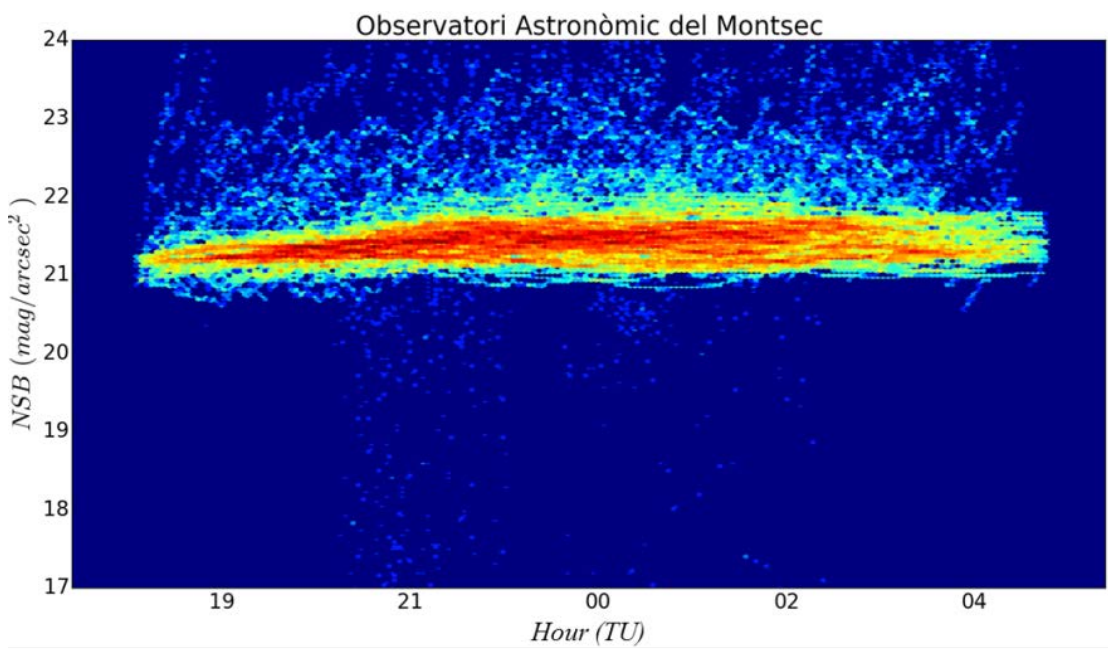

Fig. 5. Distribution of NSB data during nighttime for one year in OAdM (c. $1600 \mathrm{~m}$ asl). The data are mainly centered in 21-22 mag with scattered measurements moving towards the darkest (top) possible values. 
Opposite to what is happening in dark sky places, in Fig. 6, there is the case of Barcelona with a brighter distribution dominated by measurements around 17 mag and scattered values appearing in the bottom part of the plot, reaching extreme values up to 13-14 mag. The origin of this effect is directly linked with clouds and how they reflect artificial light at night and how clouds are amplifying ALAN effects on NSB [1, 2].

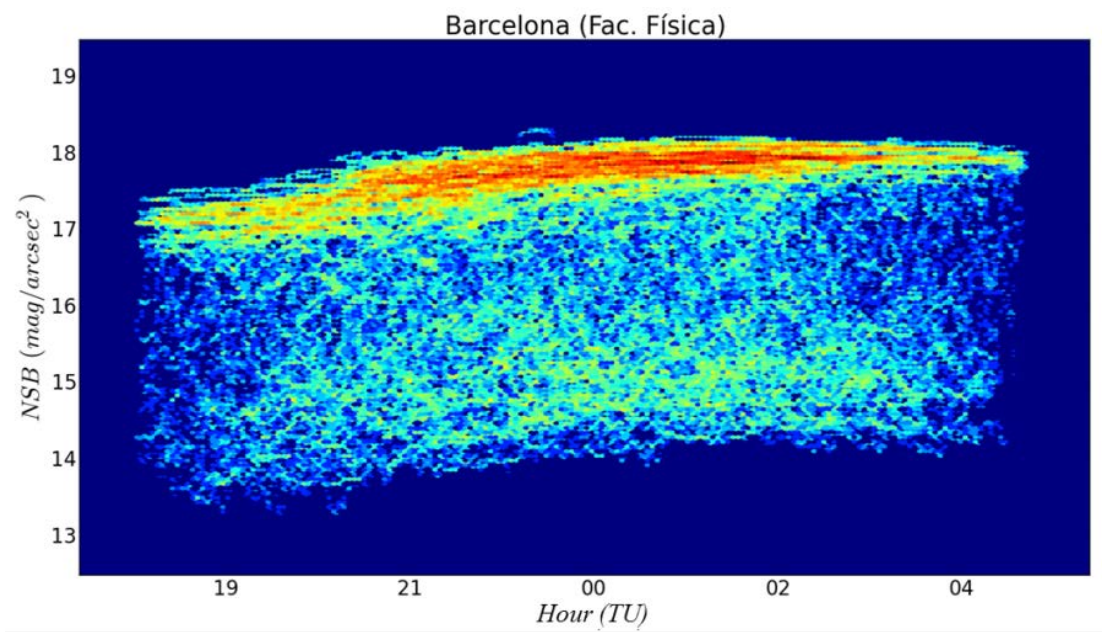

Fig. 6. Distribution of NSB data during nighttime for one year in the city of Barcelona. The data are mainly centered in 17-18 mag with scattered measurements moving towards the brightest values (13-14 mag).

Because the stations placed in Parc Astronòmic Montsec (COU and OAdM) and in Barcelona have a ceilometer device, there is available information of the presence of clouds that can be combined with NSB measurements from SQM devices. So adding available information of ceilometer data is possible to do a combined study to evaluate accurately how clouds can change NSB in both situations. The study of combined data in Barcelona city center, in the period August-November 2015, leads us to a clear example of how the clouds are amplifying the effect of LP. In the case of Barcelona, as seen in Fig.6, NSB could be six times brighter (more than 2 mag) with clouds in comparison of a clear (no clouds) night.

It is possible to compare the effect of different kinds of clouds (see Table 2). Ceilometer data provides the height of clouds and it is possible to combine NSB data, not only with presence or absence of clouds, with the levels of clouds (low, medium and high). This analysis shows how low clouds are clearly leading to the biggest increase of the effect of light in an extremely polluted area as Barcelona. In the other side high clouds are generating a tiny effect on the measurement of NSB in this city.

Table 2. Mean magnitudes of NSB measured in Barcelona with different configuration of clouds

\begin{tabular}{lccc}
\hline \multicolumn{1}{c}{ Sample } & $\begin{array}{c}\text { Number of } \\
\text { measurements }\end{array}$ & NSB & $\boldsymbol{\sigma}_{\text {mag }}$ \\
\hline & & {$\left[\mathrm{mag} / \mathrm{arcsec}^{2}\right]$} & {$\left[\mathrm{mag} / \mathrm{arcsec}^{2}\right]$} \\
\hline Total & 27645 & 16.79 & 1.21 \\
\hline Without Clouds & 14375 & 17.71 & 0.40 \\
\hline With Clouds & 13270 & 15.79 & 0.99 \\
\hline Low Clouds & 8427 & 15.53 & 0.96 \\
\hline Medium Clouds & 3643 & 16.07 & 0.84 \\
\hline High Clouds & 1200 & 16.81 & 0.63 \\
\hline
\end{tabular}

For the first time the evaluation of this effect in a natural protected area is done. The case study is Montsec mountain range as specially protected dark sky area. In this place clouds are not amplifying the effect of light. The effect is just in the opposite direction because clouds can block natural sources and NSB can be reduced to extremely dark measurements (see Fig. 5). In this case the period evaluated with ceilometer data is from November 
2014 to April 2015 and also different samples are available to evaluate NSB in different situations according to different kinds of clouds (see Table 3).

Table 3. Mean magnitudes of NSB measured in OAdM with different configuration of clouds

\begin{tabular}{lccc}
\hline \multicolumn{1}{c}{ Sample } & $\begin{array}{c}\text { Number of } \\
\text { measurements }\end{array}$ & NSB & $\sigma_{\text {mag }}$ \\
\hline Total & 66492 & {$\left[\mathrm{mag} / \mathrm{arcsec}^{2}\right]$} & {$\left[\mathrm{mag} / \mathrm{arcsec}^{2}\right]$} \\
\hline Without Clouds & 31752 & 21.47 & 0.59 \\
\hline With Clouds & 34740 & 21.44 & 0.56 \\
\hline Low Clouds & 7837 & 21.50 & 0.62 \\
\hline Medium Clouds & 15537 & 21.82 & 0.88 \\
\hline High Clouds & 11366 & 21.50 & 0.60 \\
\hline
\end{tabular}

The effect of darkening by clouds is especially important in OAdM site in the top of the mountain, without no lights in the surrounding, where low clouds sample is showing the darkest mean value, around 0.4-0.5 magnitudes darker than the sample without clouds. It is also possible to see, in Fig. 7, how the low clouds are leading to extreme NSB values (up to 24 mag) what is the zero level of SQM device.

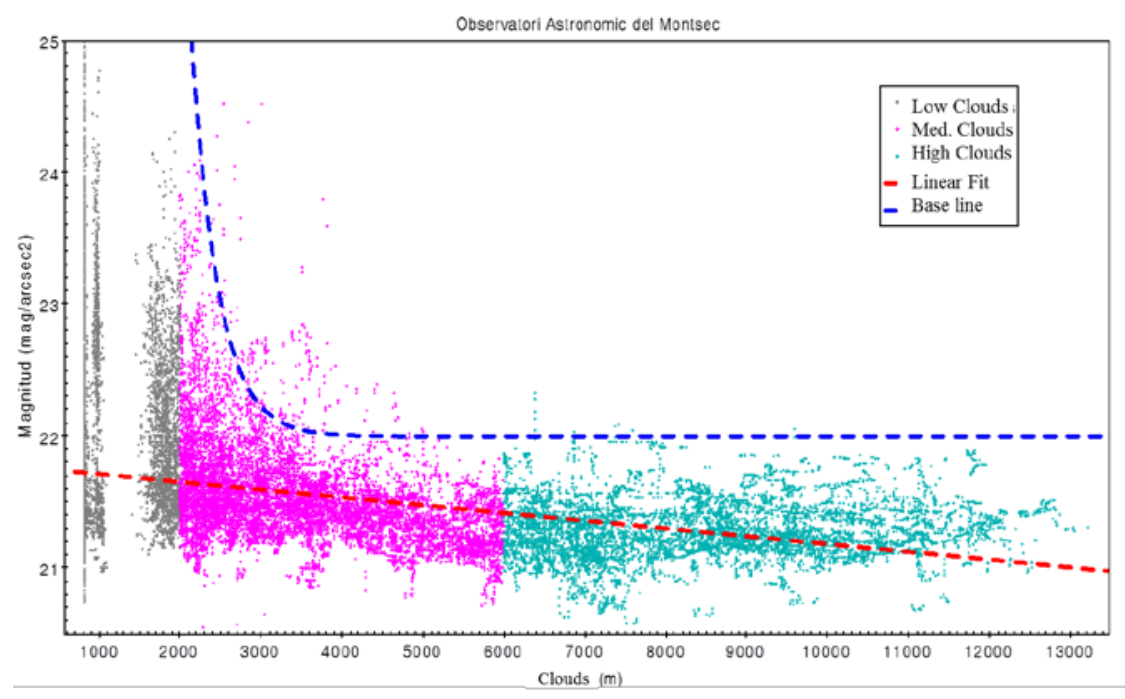

Fig. 7. Distribution of NSB measured with presence of clouds. The different subsamples are plotted and clearly low clouds can lead to darkest possible measurements of the SQM device.

\section{Conclusions}

For the first time Catalonia has a permanent network to evaluate NSB, currently it is equipped with nine stations. All the SQM devices have been previously intercalibrated. These devices are installed in a mix of protected and urban areas so comparison studies can be done. The presence of fog or clouds modifies the measurements of NSB obtained with SQM. Using only SQM data it is easy to identify intensive fog periods in the cities because fog generates a huge increase of NSB in light polluted areas. Measurements could reach 14-15 magnitudes per square arcsecond. Also without ceilometer data it is observed how in the protected areas scattered measurements are moving towards the darkest part of the plot. In the other hand in light polluted areas scattered values are appearing in the brightest parts of the plots.

For first time a combination of SQM and independent cloud data from a ceilometer has been used. With this combination of data any clouds effect is easy to determine. In the case of Barcelona (light polluted area) measurements show an increase up to 2 magnitudes of NSB due to the presence of low clouds. Also medium and high clouds have an impact increasing NSB. In the case of Montsec (protected area) clouds can darken the sky 
giving the darkest possible values of an SQM. The reason is clear, in a protected area without LP: clouds are not reflecting artificial lights and are also blocking natural features as Milky Way, Zodiacal light or stars from the sky, generating an important darkening of the sky.

\section{Acknowledgements}

Authors want to thank the institutions involved in the project of measuring and evaluating LP in Catalonia: Parc Astronòmic Montsec - Consell Comarcal de la Noguera, Institut de Ciències del Cosmos - Universitat de Barcelona (ICCUB-IEEC) and Servei per la Prevenció de la Contaminació Acústica i Lumínica (Generalitat de Caalunya). Also authors want to thank all the institutions and researchers that have provided ceilometer data: Dr. Yolanda Sola from Meteo Group of Universitat de Barcelona and Dr. Marco Pandolfi and Dr. Andres Alastuey from Institut de Diagnosi Ambiental i Estudis de l'Aigua (IDAEA) of Consejo Superior de Investigaciones Científicas.

Authors want to thank the anonymous reviewer and editors for their comments to improve this paper.

SJR acknowledges the European COST Project ES1204 Loss of the Night Network for the chance to network and improve his research on this topic.

\section{References}

[1] Longcore, T., \& Rich, C. (2004). Ecological light pollution. Frontiers in Ecology and the Environment, 2(4), 191-198.

[2] Hölker, F., Moss, T., Griefahn, B., Kloas, W., Voigt, C. C., Henckel, D., ... Tockner, K. (2010). The Dark Side of Light: A Transdisciplinary Research Agenda for Light Pollution Policy. Ecology and Society, 15(4). Retrieved from http://www.ecologyandsociety.org/vol15/iss4/art13/

[3] Gaston, K. J., Visser, M. E., Hoelker, F. (2015). The biological impacts of artificial light at night: the research challenge. Philosophical Transactions of the Royal Society of London. Series B: Biological Sciences, 370.

[4] Sánchez de Miguel, A., Zamorano, J., Castaño, J. \& Pascual, S. 2014. Journal of Quantitative Spectroscopy and Radiative Transfer 139, 109.

[5] Kyba, C. C., Ruhtz, T., Fischer, J., \& Hölker, F. (2011). Cloud coverage acts as an amplifier for ecological light pollution in urban ecosystems. PloS one, 6(3), e17307.

[6] Kyba, C. C. et al. (2015). Worldwide variations in artificial skyglow. Scientific reports, 5, 8409.

[7] Jechow, A., Hölker, F., Kolláth, Z., Gessner, M. O., \& Kyba, C. C. (2016). Evaluating the summer night sky brightness at a research field site on Lake Stechlin in northeastern Germany. Journal of Quantitative Spectroscopy and Radiative Transfer, 181, 24-32.

[8] Falchi, F., Cinzano, P., Duriscoe, D., Kyba, C. C., Elvidge, C. D., Baugh, K., ... \& Furgoni, R. (2016). The new world atlas of artificial night sky brightness. Science advances, 2(6), e1600377.

[9] de Miguel, A. S. (2015). Variación espacial, temporal y espectral de la contaminación lumínica y sus fuentes: Metodologia y resultados (Doctoral dissertation, $\mathrm{PhD}$ thesis, Universidad Complutense de Madrid).

[10] Ribas, S.J. (2015). Caracteritzacio de la Contaminacio Lumnica en Zones Protegides i Urbanes. PhD thesis, Universitat de Barcelona, Barcelona.

[11] Cinzano, P. (2005). Night sky photometry with sky quality meter. ISTIL Int. Rep, 9.

[12] Cinzano, P. (2007). Report on Sky Quality Meter, version L.

[13] Puschnig, J., Posch, T., \& Uttenthaler, S. (2014). Night sky photometry and spectroscopy performed at the Vienna University Observatory. Journal of Quantitative Spectroscopy and Radiative Transfer, 139, 64-75.

[14] Hänel, A. et al. (2017 (in preparation)). Current techniques and problems involved in measuring the night sky brightness.

[15] Nievas Rosillo, M., Zamorano Calvo, J. (2014). PySQM the UCM open source software to read, plot and store data from SQM photometers. E-prints Universidad Complutense de Madrid. Available at: http://eprints.ucm.es/25900/

[16] Bará, S. (2016). Anthropogenic disruption of the night sky darkness in urban and rural areas. Royal Society Open Science, 3(10), 160541.

[17] den Outer, P., Lolkema, D., Haaima, M., Hoff, R. V. D., Spoelstra, H., \& Schmidt, W. (2011). Intercomparisons of nine sky brightness detectors. Sensors, 11(10), 9603-9612.

[18] Pandolfi, M. et al. (2013). Continuous atmospheric boundary layer observations in the coastal urban area of Barcelona during SAPUSS. Atmospheric Chemistry and Physics, 13(9), 4983-4996. 